\title{
Free bladder mucosa graft harvested by water-jet: A novel, minimally invasive technique for urethral reconstruction
}

\author{
ZHIXIAN WANG, XIAOYONG ZENG, RUIBAO CHEN, TAO WANG, JIAHU, SHAOGANG WANG and JIHONG LIU \\ Department of Urology, Tongji Hospital, Tongji Medical College, Huazhong University of Science and Technology, \\ Wuhan, Hubei 430030, P.R. China
}

Received November 30, 2017; Accepted May 25, 2018

DOI: $10.3892 /$ etm.2018.6469

\begin{abstract}
The aim of the current study was to describe a novel approach of urethral reconstruction through minimally invasive harvesting of the bladder graft via endoscopic sub-mucosal dissection of water-jet. The records of two patients were reviewed, who underwent transurethral endoscopic surgical bladder mucosa graft harvest by water-jet and urethral reconstruction with informed consent. Case 1 was a 35 -year-old male with anterior urethral stricture; case 2 was a 22-year-old male with secondary anterior urethral stricture and hypospadias following a failed hypospadias surgery. The two male patients successfully underwent urethral reconstruction using bladder mucosa graft harvested via endoscopic assisted by water-jet; no perforation, cysthemorrhagia or any other postoperative bladder-related complication was observed. Voiding cystourethrogram of case 1 indicated that the reconstructed urethra was unobstructed, and no recrudescence was observed within 4 months of follow-up. In case 2, dysuria had disappeared completely within 1 month of follow-up, and the urethra plate was successfully reconstructed by first-stage. To the best of our knowledge, this is the first report to demonstrate urethral reconstruction using a bladder mucosa graft harvested by transurethral endoscopic sub-mucosal dissection, assisted by water-jet. Transurethral endoscopic surgery may provide a minimally invasive approach instead of the traditional open surgery for harvesting bladder mucosa graft. Urethral reconstruction conducted with bladder mucosa graft harvested via endoscopic sub-mucosal dissection assisted by water-jet is a feasible and safe method, and the short-term follow-up results are encouraging.
\end{abstract}

Correspondence to: Dr Ruibao Chen, Department of Urology, Tongji Hospital, Tongji Medical College, Huazhong University of Science and Technology, 1095 Jiefang Ave, Wuhan, Hubei 430030, P.R. China

E-mail: chen0211090@163.com

Key words: urethral stricture, reconstruction, bladder mucosa graft, water-jet

\section{Introduction}

The treatment of urethral reconstruction has changed considerably in recent decades. Although dilatation, internal urethrotomy and end-to-end anastomosis of urethral margins are the most widely used treatments, the choice of which to select should be patient-centered (1). Furthermore, these approaches are not recommended in long and complex segments of urethral stricture due to a high rate of failure (1). In recent decades, free grafts including free skin (penile, scrotal, extragenital) flap, bladder mucosa, oral (buccal and lingual) mucosa and colonic mucosa, have been successfully applied to urethroplasty (2). Of these, oral mucosa graft is considered to be an ideal substitute for the urethra, with high success rates because of its characteristics of resistance to infection and compatibility with a wet environment (3). Although oral mucosa grafts are now widely applied in urethroplasty (2), they are associated with certain early and late postoperative complications, including oral pain, mouth tightness, speech and chewing difficulties, altered sensation and reduced tongue protrusion. Certain patients are unsuitable for undergoing urethroplasty with oral mucosa graft due to difficulties with harvesting sufficient oral mucosa $(2,3)$. Therefore, alternative grafts are important. Since bladder mucosa was first used for urethra reconstruction by Memmelaar in 1947 (4), it has been demonstrated to be feasible and safe in urethral reconstruction in several cases (4-7). Therefore, bladder mucosa represents alternative graft material in certain patients who are not candidates for performing oral mucosa graft harvest.

To harvest bladder mucosa graft, patients undergo conventional open surgery, which may be followed by numerous complications (6,7). In the current study, a novel, minimally invasive method is reported that has been applied to harvest bladder mucosa graft though an endoscopic technique assisted by water-jet. The safety, feasibility and efficacy of this approach are discussed.

\section{Patients and methods}

Case data. Case 1 was a 35-year-old male patient with a complaint of dysuria for 3 years. The patient was admitted to the Department of Urology, Tongji Hospital (Wuhan, China) in April 2017. He had a history of straddle injury 3 years previously, and had undergone internal urethrotomy twice 

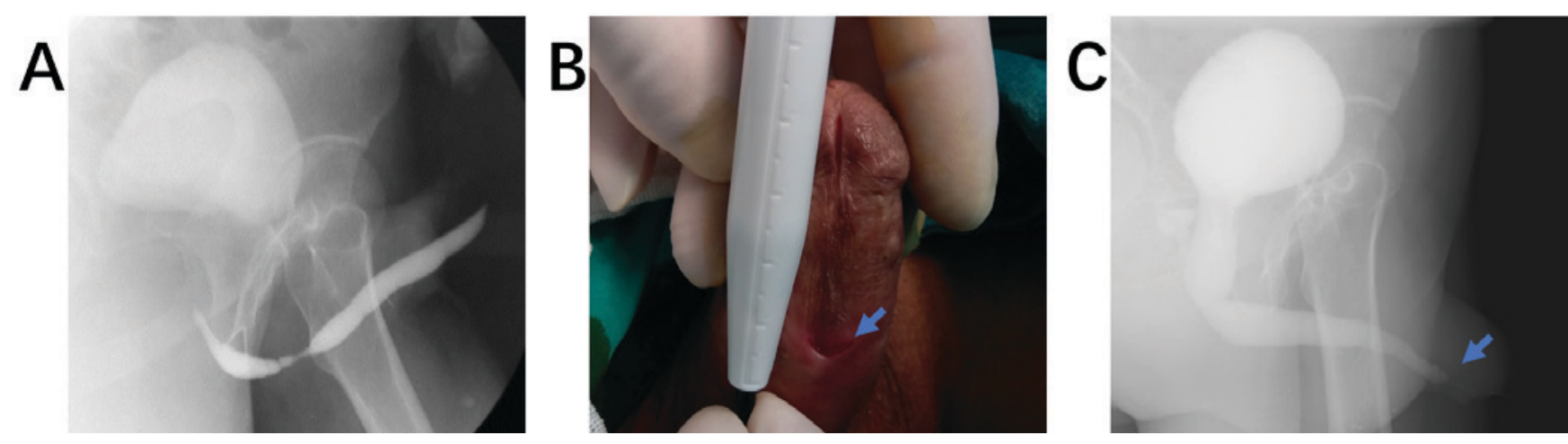

Figure 1. Pathological changes and imaging data. (A) Voiding cystourethrogram in case 1 preoperative. (B) Urethral orifice was located at the middle of the penis in case 2. (C) Voiding cystourethrogram in case 2 preoperative.

and numerous instances of urethral dilatation for the injury. Urethrography suggested that a bulbar urethral stricture was present (Fig. 1A). At first, lingual mucosa was recommended for urethral reconstruction. However, the patient rejected this treatment once he understood the advantages and disadvantages associated with urethral reconstruction with oral mucosa. Eventually, urethral reconstruction with bladder mucosa graft harvested via endoscopic sub-mucosal dissection assisted by water-jet was adopted.

Case 2 was a 22-year-old male patient who had suffered from dysuria for 1 year, in the absence of fever or discomfort of the waist and abdomen. The patient was admitted to the Department of Urology, Tongji Hospital (Wuhan, China) in May 2017. He had undergone urethroplasty surgery for hypospadias in 2001 and 2008. Physical examination indicated that the urethral orifice was located at the middle of the penis (Fig. 1B). Preoperative cystography indicated a segment of urethral stricture of $\sim 1.5 \mathrm{~cm}$ (Fig. 1C). There was no residual urine under bladder ultrasonography (Table I).

The two patients provided written, informed consent for their inclusion in the current study.

Bladder mucosa graft harvest. The patients received ultrasonography and cystoscopy to exclude urinary system diseases, including inflammatory and neoplasm conditions, which would preclude harvest bladder mucosa grafts. Routine urine examination, as well as urine culture, was also necessary to determine whether urinary tract infection was present.

The patient received general anesthesia and was placed in the lithotomy position. The stricture was searched under the guidance of catheter. The skin, the perineal superficial and deep fascia and the corpus spongiosum were dissected layer by layer. Before bladder mucosa was harvested, the length of the stricture segment or position of hypospadias was measured, and then the endoscope was placed into the bladder from the normal urethra under direct vision (Fig. 2). Subsequently, the surgical procedure of endoscopic sub-mucosal dissection was performed using the water-jet hybrid knife system (8) (I-Type; diameter, $2.3 \mathrm{~mm}$; length $1.9 \mathrm{~m}$; Erbe China Ltd., Shanghai, China; Fig. 3).

The detailed endoscopic surgical procedure (Fig. 4) was as follows: Boundaries of resection were marked by a series of electrical coagulation points; a sub-mucosal 'water pad' was formed by the high-pressure water column penetrating the mucous layer and gathering under the mucous membrane to separate the mucosa and muscle layer; the mucosa membrane was dissected along the edge of the marker points; active bleeding was stopped via electrocoagulation and the bladder mucosa graft was obtained.

Urethral reconstruction technique. The bladder mucosa graft was soaked in normal saline at room temperature for $20 \mathrm{~min}$ prior to use. The thicker mucosa was thinned and trimmed according to the size required (Fig. 5A). Dorsal onlay urethroplasty was performed for case 1 (Fig. 5B and C) and urethra plate reconstruction was performed for case 2 . The caliber of the reconstructed urethra should be spacious enough to insert the $22 \mathrm{Fr}$ three cavity catheter for continuous bladder irrigation postoperative. The incision was sewn up layer by layer. Throughout the whole process, physiological saline and dilute iodine were used to maintain a moist condition for the wound, and electrocoagulation was generally not recommended except for massive bleeding.

Bladder irrigation was stopped once hemorrhaging disappeared. The catheter was removed 3-4 weeks later, then voiding cystourethrogram was performed to check urethral condition.

\section{Results}

The surgeries were conducted successfully. One-stage urethral reconstruction was performed in case 1 , and the urethra plate was reconstructed by first-stage in case 2 (Fig. 5D). No perforation, active cysthemorrhagia or any other complication occurred during or following surgery. Case 1 had unobstructed urination once the catheter was removed at 1 month following surgery, and voiding cystourethrogram indicated that the reconstructed zone was normal (Fig. 5E). Within 4 months of follow-up, there was no recrudescence. Case 2 is currently waiting for the second-stage urethral reconstruction. The urethra plate reconstructed by bladder mucosa graft has survived, and dysuria has disappeared.

\section{Discussion}

Currently, urethral reconstruction with oral mucosa graft is the most widely performed surgical approach in urethroplasty, which has numerous advantages (2). These include 
Table I. Clinical and surgical characteristics.

A, Clinical characteristics

\begin{tabular}{lcc}
\hline Characteristic & Patient 1 & Patient 2 \\
\hline Age (years) & 35 & 22 \\
Sex & Male & Male \\
BMI $\left(\mathrm{kg} / \mathrm{m}^{2}\right)$ & 22.0 & 23.9 \\
Medical history & Straddle injury; internal urethrotomy twice and & Hypospadias; two urethroplasty \\
& numerous instances of urethral dilatation & surgeries for hypospadias \\
Lesion location & Bulbous urethra & Penile urethra \\
Length of stricture $(\mathrm{cm})$ & 2.0 & 1.5 \\
Urethral orifice & Normal & Scrotum \\
\hline
\end{tabular}

B, Surgical characteristics

\begin{tabular}{lcc}
\hline Characteristic & Patient 1 & Patient 2 \\
\hline Stage of urethroplasty & One-stage approach & Two-stage approach \\
Onlay of free bladder mucosal & Dorsal onlay & - \\
Operative time (min) & 167 & 156 \\
Operative time of bladder mucosal & 35 & 30 \\
harvest (min) & 120 & 100 \\
Blood loss (ml) & No & No \\
Severe complications & & \\
\hline
\end{tabular}

BMI, body mass index.

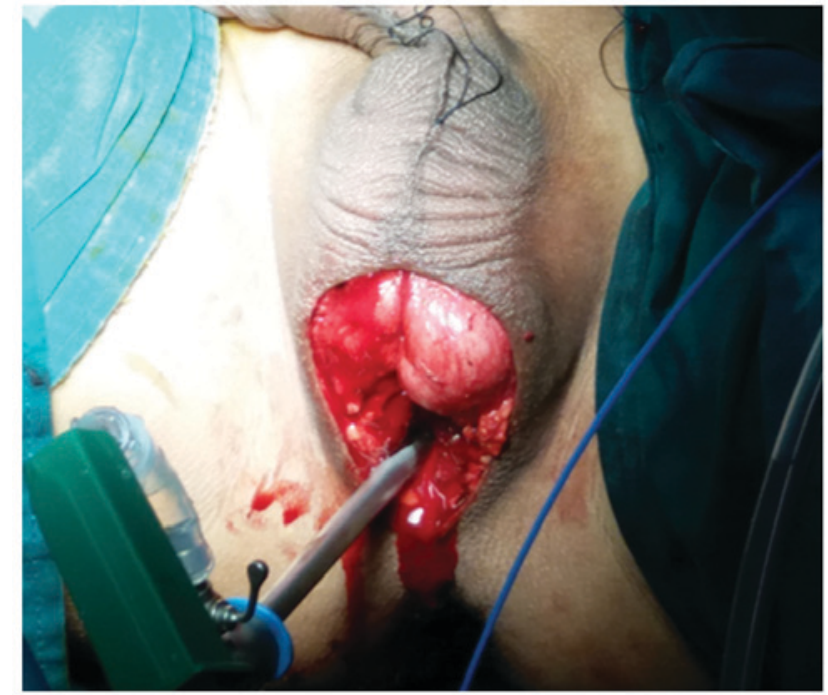

Figure 2. Surgical procedure. The endoscope was placed into the bladder through the normal posterior urethra under direct vision, following dissection of the narrow segment.

the relative ease of harvesting oral mucosa and minimal complications that may occur. However, the drawbacks are the limited availability of tissue and donor site morbidity $(2,9,10)$. Alternative grafts are also required for patients who are not candidates for performing oral mucosa graft harvest, particularly for long, narrow segments and for recurrent strictures following prior operation using oral mucosa graft. Xu et al (11) reported a feasible procedure for complex urethral strictures using circumferential colonic mucosa grafts, which achieved a high success rate of $86 \%$. Palmer et al (12) reported a feasible, safe and low morbidity technique for harvesting rectal mucosa graft for urethral reconstruction via a transanal endoscopic microsurgical technique. However, this technique carries a risk of colonic perforation and requires an experienced surgeon, as well as absence of bowel disease in the patient, which limit the extensive use of rectal mucosa grafts among many institutions. To the best of our knowledge, this is the first report of using bladder mucosa graft harvested via endoscope assisted by water-jet for urethral reconstruction, which is a minimally invasive approach compared with conventional surgeries.

Bladder mucosa, with the advantage of providing long segments, was first used for urethra reconstruction by Memmelaar in 1947 (4). Bladder mucosa is easy to obtain and does not usually affect bladder function postoperatively. For the current cases, recovery time was short following the minimal invasive surgery. Bladder mucosa may be used for long segment urethral stricture reparation, since it is easy to obtain long segments of bladder mucosa. Furthermore, bladder mucosa has similar characteristics to urethral epithelium (2,5-7,13). Although complications, including meatal stenosis, prolapse and shrinkage of bladder mucosa, may occur, a high success rate could be achieved by selecting suitable cases $(6,7)$. Follow-up results in the current study 
A

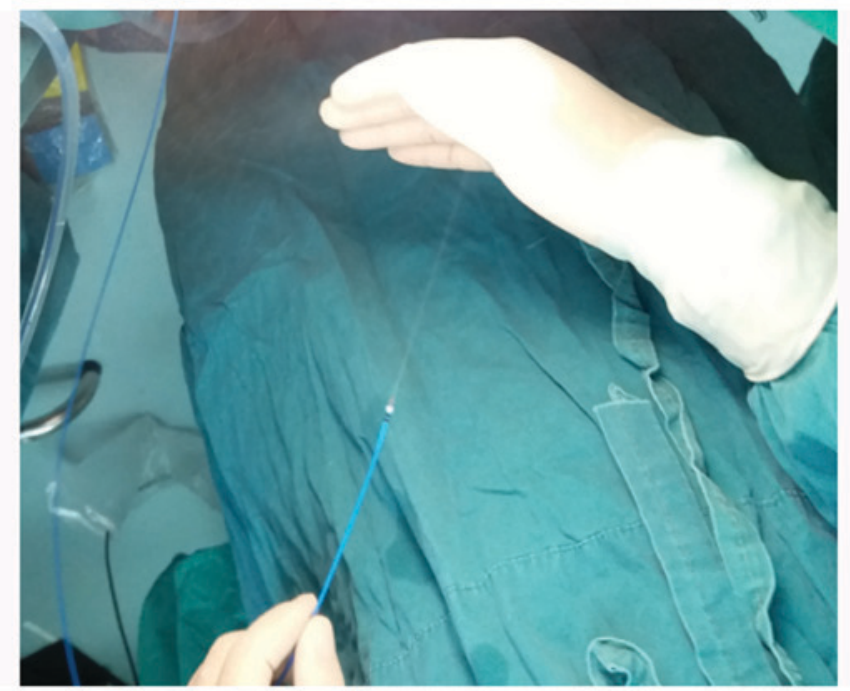

B

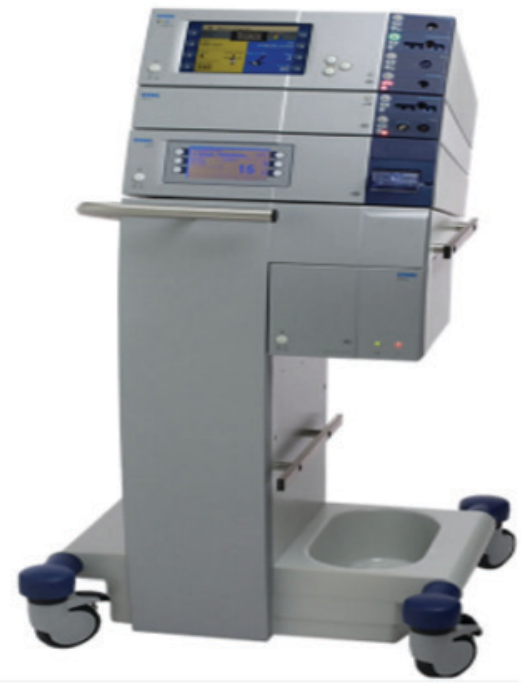

Figure 3. Water-jet hybrid knife system. (A) Water-jet (I-Type, Ø2.3 mm, length 1.9 m). (B) Hybrid knife system (Erbe China Ltd., Shanghai, China).

A

D
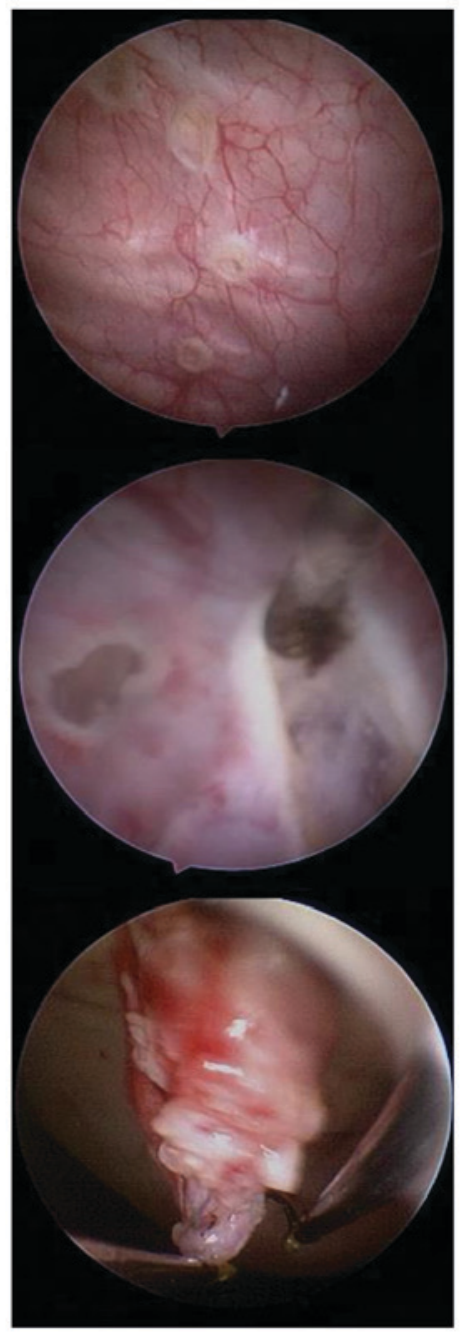

B

$\mathrm{E}$
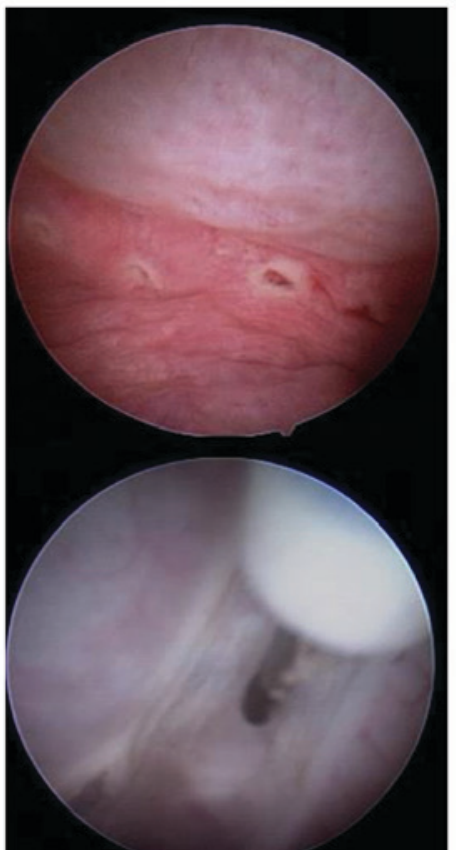

$\mathrm{H}$

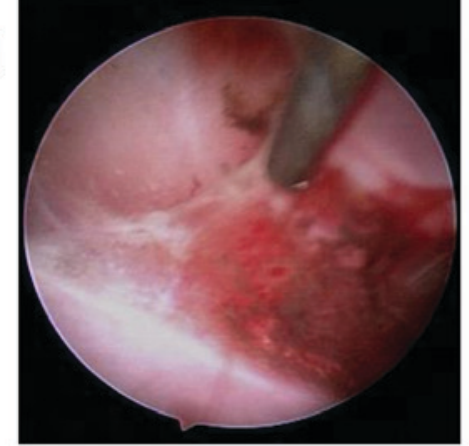

C

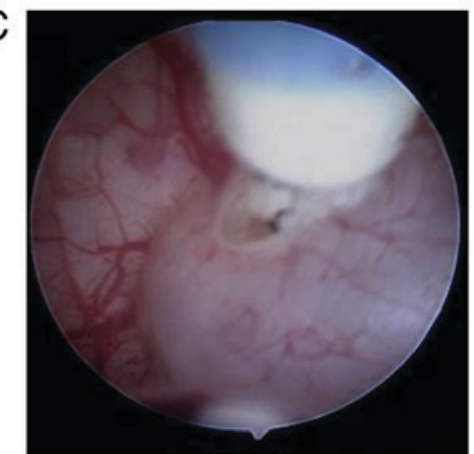

F

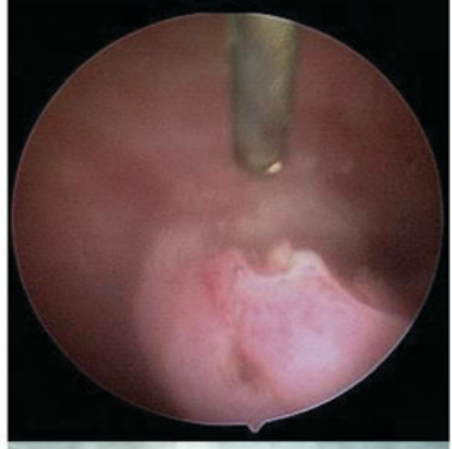

।

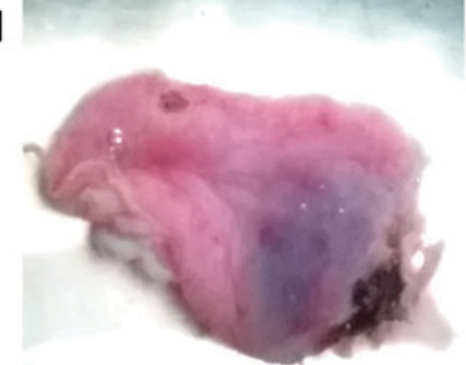

Figure 4. Bladder mucosa graft harvest. (A) Marking the edges of mucosal resection. (B) Forming sub-mucosal 'water pad' to separate the mucosa and muscle layer. (C-G) Resection of bladder mucosa graft assisted by water-jet. (H) Complete hemostasis. (I) Acquired bladder mucosa graft.

suggest that the approach is feasible and safe. Short-term successful outcomes have also been described previously $(4,6,12)$. In order to avoid the potential complications following conventional open surgery for bladder mucosa graft harvest, patients in the current study underwent a minimally invasive approach of transurethral endoscopic sub-mucosal 

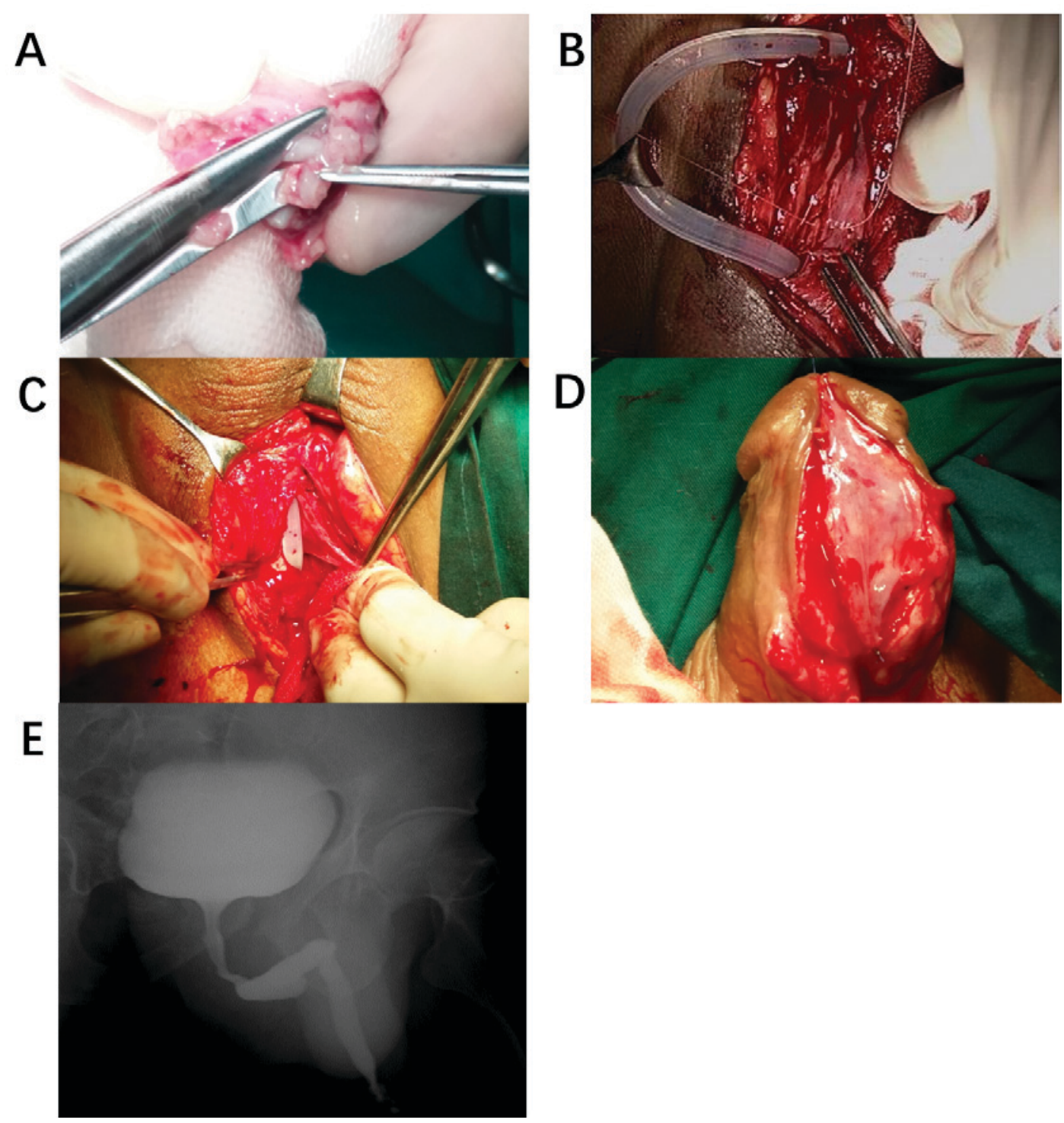

Figure 5. Urethral reconstruction with bladder mucosa. (A) The bladder mucosa was thinned and trimmed to the size required. (B and C) Dorsal onlay urethroplasty in case 1. (D) Urethra plate reconstruction in case 2. (E) Voiding cystourethrogram in case 1 at 1 month after urethral reconstruction with bladder mucosa.

dissection via water-jet to harvest the bladder mucosa. This technique is performed on the bladder, which is familiar to a urologist and easy to operate on, and is relatively quick to learn (8). This novel minimally invasive technique could potentially be provided to patients who are not suitable candidates for oral mucosa graft.

The technique of endoscopic sub-mucosal dissection assisted by water-jet in the treatment of digestive tract neoplasia has been widely used in the clinic, and has been demonstrated to be a safe and effective method in a minimally invasive operation $(8,14)$. Fritsche et al $(8)$ presented the first prospective clinical trial on the application of the technique in transurethral dissection of the large bladder carcinoma in 2011. Compared with the digestive tract and colon, the bladder mucosa and muscle layer are thicker, which makes the surgery safer, with a low incidence of vesical perforation and obturator nerve reflex). Furthermore, bladder mucosa is less exposed to electrical damage and can be kept alive more successfully once it is removed from the bladder (8).
In terms of postoperative bleeding, complete hemostasis intra-operative is critical for the success of reconstructive surgery, because reoperation may destruct the operative area. According to the data collected from patients who underwent sub-mucosal dissection of bladder tumor via water-jet, (which has a similar procedure to the harvest of bladder mucosa in the present study), no cases of postoperative bleeding were identified (8).

In conclusion, urethral reconstruction using bladder mucosa graft harvested via transurethral endoscopic sub-mucosal dissection assisted by water-jet is an alternative technique for patients who are not deemed as suitable candidates for urethroplasty with oral mucosa graft. However, further study of cases with longer follow-up periods are required to demonstrate its safety and feasibility.

\section{Acknowledgements}

Not applicable. 


\section{Funding}

The present study was supported by Research Funds of Tongji Hospital, HUST and Natural Science Foundation of Hubei Provence to RC (grant no. 2017CFB714). This study was also supported by Key Items of Health and Family Planning Commission of Hubei Province to XZ (grant no. WJ2015MA015)

\section{Availability of data and materials}

Data sharing is not applicable to this article, as no datasets were generated or analyzed during the current study.

\section{Authors' contributions}

ZW, RC and XZ conceived and designed the present study; RC and $\mathrm{JH}$ performed the experiments; TW, JL and SW analyzed data; RC and ZW interpreted the results; ZW and RC prepared the figures; and ZW, RC and XZ edited and revised manuscript. All authors approved final version of manuscript.

\section{Ethics approval and consent to participate}

Ethical approval was granted by the medical ethics committee of Tongji hospital, Tongji medical college, Huazhong University of Science and Technology (Wuhan, China).

\section{Patient consent for publication}

Written informed consent was obtained from the patients for the publication of this article and any accompanying images. The information used to identify patients was deleted.

\section{Competing interests}

The authors declare that they have no competing interests.

\section{References}

1. Mangera A and Chapple C: Management of anterior urethral stricture: An evidence-based approach. Curr Opin Urol 20: 453-458, 2010.

2. Mangera A, Patterson JM and Chapple CR: A systematic review of graft augmentation urethroplasty techniques for the treatment of anterior urethral strictures. Eur Urol 59: 797-814, 2011.

3. Bhargava S and Chapple CR: Buccal mucosal urethroplasty: Is it the new gold standard? BJU Int 93: 1191-1193, 2004.

4. Memmelaar J: Use of bladder mucosa in a one-stage repair of hypospadias. J Urol 58: 68-73, 1947.

5. Monfort G, Bretheau D, Di Benedetto V and Bankole R: Urethral stricture in children: Treatment by urethroplasty with bladder mucosa graft. J Urol 148: 1504-1506, 1992.

6. Kinkead TM, Borzi PA, Duffy PG and Ransley PG: Long-term followup of bladder mucosa graft for male urethral reconstruction. J Urol 151: 1056-1058, 1994.

7. Ozgok Y, Ozgür Tan M, Kilciler M, Tahmaz L and Erduran D: Use of bladder mucosal graft for urethral reconstruction. Int $\mathrm{J}$ Urol 7: 355-360, 2000.

8. Fritsche HM, Otto W, Eder F, Hofstädter F, Denzinger S, Chaussy CG, Stief C, Wieland WF and Burger M: Water-jet-aided transurethral dissection of urothelial carcinoma: A prospective clinical study. J Endourol 25: 1599-1603, 2011.

9. Chapple C, Andrich D, Atala A, Barbagli G, Cavalcanti A, Kulkarni S, Mangera A and Nakajima Y: SIU/ICUD consultation on urethral strictures: The management of anterior urethral stricture disease using substitution urethroplasty. Urology 83 (Suppl 3): S31-S47, 2014.

10. Barbagli G, Vallasciani S, Romano G, Fabbri F, Guazzoni G and Lazzeri M: Morbidity of oral mucosa graft harvesting from a single cheek. Eur Urol 58: 33-41, 2010.

11. Xu YM, Qiao Y, Sa YL, Zhang J, Fu Q and Song LJ: Urethral reconstruction using colonic mucosa graft for complex strictures. J Urol 182: 1040-1043, 2009.

12. Palmer DA, Marcello PW, Zinman LN and Vanni AJ: Urethral reconstruction with rectal mucosa graft onlay: A novel, minimally invasive technique. J Urol 196: 782-786, 2016.

13. Wessells $\mathrm{H}$ and McAninch JW: Use of free grafts in urethral stricture reconstruction. J Urol 155: 1912-1915, 1996.

14. Schumacher B, Charton JP, Nordmann T, Vieth M, Enderle M and Neuhaus H: Endoscopic submucosal dissection of early gastric neoplasia with a water jet-assisted knife: A Western, single-center experience. Gastrointest Endosc 75: 1166-1174, 2012. 